\section{Fatores de risco e preditores para o aborto induzido: estudo de base populacional}

\author{
Risk factors and predictors of induced abortion: \\ a population-based study
}

1 Programa de Pós-graduação
em Saúde Coletiva,
Universidade do Vale
do Rio dos Sinos,
São Leopoldo, Brasil.
2 Faculdade de Ciências
Médicas, Universidade
Estadual de Campinas,
Campinas, Brasil.
Correspondência
M. T. A. Olinto
Programa de Pós-graduação
em Saúde Coletiva,
Universidade do Vale
do Rio dos Sinos.
Av. Unisinos 950, C. P. 275,
São Leopoldo, RS
93022-00, Brasil.
mtolinto@unisinos.br

Abstract

This study aimed to identify key risk factors and predictors of induced abortion. A cross-sectional population-based study was conducted with a representative sample of 3,002 women 15 to 49 years of age in southern Brazil, randomly assigned to answer questions on induced abortion using either the ballot-box method or the indirect questioning method. Socioeconomic, demographic, and reproductive data were obtained through a pre-coded questionnaire. Data analysis used epidemiological statistical inferences and Bayes' theorem to calculate a posteriori probability. Induced abortion was strongly associated with fetal loss for all age groups. In adolescents, the main predictors were low socioeconomic level, low schooling, elevated school drop-out, and knowledge of a large number of contraceptive methods. For all other women, socioeconomic characteristics and skin color were not associated with abortion. For women aged 20 to 49 years, marital status and reproductive characteristics, including knowledge of contraceptive methods, were the most frequent risk factors and predictors of induced abortion.

Women's Health; Induced Abortion; Risk Factors
Maria Teresa Anselmo Olinto 1

Djalma de Carvalho Moreira-Filho ${ }^{2}$

A Organização Mundial da Saúde estima que, no mundo todo, cerca de 500 mil mulheres morrem a cada ano de causas relacionadas à gestação, sendo que $98 \%$ dessas ocorrem nos países em desenvolvimento. Nesses locais, complicações de aborto são responsáveis por $15 \%$ do total das mortes maternas a cada ano - em alguns casos, atinge cerca de $50 \%$ dessa mortalidade 1 . Considera-se aborto induzido, ou aborto provocado, o término da gestação devido à interferência deliberada antes que o feto seja capaz ter vida extra uterina, e inclui aqueles previstos ou não por lei.

Em geral, países com leis restritivas ao aborto apresentam altas taxas de aborto inseguro 2 . No caso do Brasil, o Código Penal inclui o aborto entre os crimes contra a vida e prevê duas exceções: nos casos de estupro e de risco para a vida da mãe. A ilegalidade do aborto não tem impedido que ele ocorra indiscriminadamente entre as diferentes classes sociais no Brasil, mas, certamente, o fato de ter ou não complicações pós-aborto é sócio e economicamente dependente. Enquanto mulheres de classes sociais mais privilegiadas recorrem ao aborto em clínicas privadas com procedimentos seguros, mulheres pertencentes a classes sociais menos favorecidas são expostas a procedimentos inseguros 3 , na maioria das vezes, realizados por profissionais não especializados utilizando-se 
de técnicas perigosas que podem acarretar risco de vida ou seqüelas irreversíveis 4 .

O aborto induzido é um sério problema de saúde pública que ficou um longo período alijado dos debates dos diversos segmentos da sociedade e, principalmente, das decisões políticas neste país 5. Recentemente, o debate da legalização do aborto ganhou espaço na mídia devido à norma do Ministério da Saúde que autoriza os médicos da rede pública a fazer aborto em mulheres que aleguem ter engravidado por estupro, mesmo sem a apresentação do boletim de ocorrência policial.

Neste contexto, demógrafos, epidemiologistas, sociólogos, antropólogos, entre outros pesquisadores, têm o compromisso de fornecer informações para subsidiar os debates atuais e futuros. Portanto, faz-se urgente a realização de investigações que estabeleçam a real magnitude do aborto induzido no Brasil e identifiquem os fatores de risco envolvidos.

Diversos métodos de investigação são propostos para o tema, dependendo do contexto e da finalidade específica da investigação 6 . Embora existam vários estudos sobre o aborto induzido, ainda são poucos os que utilizam amostras representativas, de base populacional e que visem a identificar fatores de risco, especialmente neste contexto de ilegalidade.

\section{Metodologia}

\section{Desenho}

Foi realizado um estudo transversal de base populacional com uma amostra representativa de 3.002 mulheres em idade reprodutiva ( 15 a 49 anos), residentes na zona urbana de Pelotas, Rio Grande do Sul, Brasil. Este estudo foi desenhado para atender dois objetivos: (1) comparar duas metodologias para estimar a freqüência de aborto induzido na população; e (2) identificar fatores de risco e preditores para o aborto induzido em mulheres de 15 a 49 anos. Para atender cada um desses objetivos foram desenvolvidas diversas etapas de investigação. O detalhamento dos métodos para atingir o primeiro objetivo encontra-se em outra publicação 7 . No presente artigo, encontram-se especificados materiais e métodos utilizados para atingir o segundo objetivo.

\section{Amostra}

Para o cálculo do tamanho da amostra, vários ensaios foram realizados com base nas estimativas de prevalência de aborto induzido em es- tudos de base populacional (4 a 10\%), com um poder estatístico de 80 a $90 \%$, um erro alfa de $5 \%$ e uma razão de expostos para não-expostos de 1:1 - devido à alocação para cada uma das duas metodologias utilizadas na investigação do primeiro objetivo acima citado. Após algumas simulações, o tamanho de amostra escolhido foi de 3.098, com uma probabilidade de $80 \%$ de detectar uma razão de prevalências de 1,5 para uma prevalência de abortos em nãoexpostos de $5 \%$. Prevendo-se possíveis perdas e recusas, o tamanho de amostra foi aumentado para 3.200 mulheres.

Para alcançar esse total de mulheres e sabendo-se que nessa faixa etária encontra-se cerca de 0,92 mulher/domicílio, seria necessário visitar cerca de 3.478 domicílios. Portanto, visitando setenta domicílios em cada um dos cinqüenta setores censitários (entre os 258 existentes na cidade), seriam encontradas em média 64 mulheres por setor, atingindo o tamanho de amostra calculado. A seleção dos cinqüenta setores censitários foi feita por meio de uma amostragem sistemática, o que garantiria que as diferentes áreas da cidade fossem representadas - e, portanto, os diversos grupos sócio-econômicos. O passo seguinte no processo amostral foi a escolha do local em cada setor onde seria iniciado o trabalho de campo. Para isso, mapas individuais dos setores censitários foram desenhados e todas as quadras numeradas. Para cada um dos mapas, sorteava-se a quadra inicial do trabalho e, nessa quadra, a esquina inicial. Uma vez definido o ponto de partida, as entrevistadoras eram orientadas a caminhar sempre para a mesma direção, a fim de evitar viés de seleção, tal como entrevistar apenas as mulheres que morassem em casas de mais fácil acesso.

\section{Instrumentos}

As informações sócio-econômicas, demográficas e reprodutivas das mulheres da amostra foram coletadas por meio de um questionário geral aplicado por entrevistadoras a cada uma das mulheres da amostra. As variáveis demográficas incluíram: idade, cor da pele/etnia (observada pela entrevistadora), religião e estado civil. As variáveis sócio-econômicas incluíram: escolaridade da mulher, renda familiar, atividade atual (estuda e/ou trabalha e/ou donade-casa) e domicílio com abastecimento de água e/ou rede de esgoto. As informações sobre a vida reprodutiva das mulheres foram coletadas por meio das seguintes variáveis: vida sexual ativa ( $\operatorname{sim} /$ não), uso de métodos contraceptivos, problemas no uso de métodos con- 
traceptivos, esterilização (sim/não), conhecimento de métodos contraceptivos, número de gestações, número de filhos (nascidos vivos), desejo de ter mais filhos (sim/não) e perda fetal. A perda fetal foi avaliada por meio de variáveis: (1) variável "perda fetal" obtida por uma pergunta direta à mulher sobre ocorrência na vida de alguma gravidez em que o filho tenha nascido morto; (2) variável "perda fetal $<7$ m" para as perdas ocorridas com menos de sete meses de gestação; e (3) variável "perda fetal I" informação de perda fetal obtida de forma indireta, ou seja, através da subtração entre o relato do número de gestações e o número de filhos tidos, excluindo-se aqueles que faleceram após o nascimento. Todas as perguntas referentes à perda fetal, bem como as outras variáveis que descreviam as características sócio-econômicas, demográficas e reprodutivas foram coletadas antes das informações específicas de aborto induzido.

As informações sobre aborto induzido foram obtidas utilizando-se duas metodologias diferentes, o método da urna e o método das questões indiretas. O detalhamento desses instrumentos e os resultados da comparação das estimativas de aborto obtidos em cada um deles encontram-se em outra publicação 7 . Resumidamente, no método da urna as mulheres colocavam um "voto" sem identificação pessoal, apenas com informações sobre a ocorrência de aborto induzido e a sua idade. Todas as urnas eram identificadas por setor censitário, permitindo que as informações de aborto fossem agregadas por cada um dos cinqüenta setores sorteados. O outro método, o das questões indiretas, investigava aborto por meio de uma pergunta indireta sobre gravidez indesejada aplicada a cada uma das mulheres, permitindo a informação no nível individual. Mais detalhes sobre a estrutura desse método podem ser encontrados nas publicações de Huntington et al. 8 e Olinto ${ }^{9}$.

\section{Entrevistas}

Todas as entrevistas foram realizadas nos domicílios por entrevistadoras, todas mulheres, maiores de 18 anos de idade e estudantes universitárias da área de ciências humanas e da saúde, rigorosamente treinadas.

No início de cada setor era feito o sorteio de qual método - método da urna ou das questões indiretas - a entrevistadora deveria utilizar para a primeira mulher a ser entrevistada. A partir do primeiro método sorteado, da urna ou das questões indiretas, as próximas mulheres em cada setor eram alocadas alternadamente para um ou outro método. O questionário geral era aplicado no início de cada entrevista e as perguntas sobre aborto eram realizadas ao final da entrevista.

\section{Controle de qualidade}

Para assegurar o controle de qualidade das informações foram refeitas $5 \%$ das entrevistas utilizando-se um questionário simplificado. A codificação dos questionários foi realizada pelas entrevistadoras e revisada pela coordenação da pesquisa. A digitação dos dados seguiu o procedimento de dupla entrada no programa Epi Info (Centers for Disease Control and Prevention, Atlanta, Estados Unidos), comparação de digitações e análise de consistência.

\section{Análise dos dados}

A análise dos dados, realizada no SPSS 8.0 (SPSS Inc., Chicago, Estados Unidos), constituiu-se de: análise bivariada entre as variáveis socioeconômicas, demográficas e reprodutivas com a variável aborto (nível de significância $\mathrm{p}<0,05$ ); cálculo da probabilidade $a$ posteriori com o objetivo de otimizar as informações de aborto por meio da transformação de dados agregados do método da urna para individuais; análise multivariada por meio de regressão logística controlando para variáveis sócio-econômicas e demográficas que apresentaram significância estatística com $\mathrm{p}<0,05$; e análise estratificada por idade para descrever os fatores de risco e preditores de aborto.

\section{Aspectos éticos}

O projeto desta pesquisa foi aprovado pelo Comitê de Ética e Pesquisa da Universidade Federal de Pelotas; todas as mulheres participaram livremente do estudo e foram informadas dos passos e objetivos da pesquisa; no banco de dados não foram ingressadas informações de identificação pessoal; após a digitação das informações as identificações pessoais contidas nos questionários foram apagadas; e todo o processo que envolveu a passagem de dados agregados para o nível individual foi realizado com o total anonimato das mulheres, apenas utilizando-se de números.

\section{Resultados}

Com base nos cinqüenta setores censitários sorteados para o estudo, 3.857 domicílios foram visitados e 3.154 mulheres de 15 a 49 anos 
localizadas. Após recusas e perdas (impossibilidade de contatar depois de três tentativas), 3.002 mulheres foram incluídas no estudo. O cálculo da estimativa inicial de 3.200 mulheres estava superestimado, ao invés de serem encontradas 64 mulheres de 15 a 49 anos, em setenta domicílios, foram encontradas, em média, sessenta mulheres.

Todas as 3.002 mulheres responderam o questionário geral para as informações sócioeconômicas, demográficas e reprodutivas. Para as informações sobre aborto, 1.510 responderam pelo método das questões indiretas e 1.492 pelo método da urna. A diferença de 18 mulheres foi devido ao processo de alocação para um ou para o outro método ter sido realizado dentro de cada setor censitário.

As 3.002 mulheres tinham em média 31 anos de idade (IC95\%: 30,6-31,4), 84\% eram brancas, $56 \%$ eram casadas ou viviam em união, $59 \%$ consideravam-se católicas, $50 \%$ tinham trabalho remunerado, tinham escolaridade média de 8,5 anos (IC95\%: 8,3-8,6) e 31\% viviam com renda familiar igual ou inferior a três salários mínimos. Quanto às condições de moradia, $84 \%$ das casas apresentavam sanitários com descarga e, $96 \%$ água encanada no seu interior (dados não apresentados em tabela).

No total da amostra, 165 mulheres relataram ter provocado aborto, sendo 58 relatos pelo método das questões indiretas e 107 pelo método das urnas, respectivamente, uma ocorrência de $3,8 \%$ e $7,2 \%$. O relato de aborto apresentou um aumento linear com a idade das mulheres, $1,6 \%, 3,1 \%, 6,5 \%$ e $9 \%$, respectivamente, para os grupos de 15 a 19 anos, 20 a 29, 30 a 39 e 40 a 49 anos de idade $(\mathrm{p}<0,001)$. Todas as outras variáveis presentes no questionário geral, que poderiam ser preditores ou fatores de risco para o desfecho, foram testadas utilizando-se de análises bivariadas no grupo de mulheres do método das questões indiretas. As variáveis estado civil, religião, conhecimento de métodos contraceptivos, problemas com uso de métodos contraceptivos, desejo de ter mais filhos e perdas fetais apresentaram associação estatisticamente significativa ( $p \leq 0,05)$. Nenhuma das variáveis sócio-econômicas apresentou associação estatisticamente significativa com aborto induzido. Também não foi observada diferença no relato de aborto segundo a cor da pele. Observou-se que, quase a totalidade das mulheres com aborto induzido tiveram perdas fetais.

A Tabela 1 detalha a associação de perda fetal e relato de aborto induzido para as mulheres selecionadas para o método das questões indiretas. De acordo com a variável "perda fetal", 94,8\% das mulheres com aborto induzido relataram ter um filho que nasceu morto, sendo que todos com idade gestacional menor do que sete meses. No grupo de mulheres que não abortaram apenas $13 \%$ relataram perda fetal. Por meio da variável perda fetal avaliada de forma indireta ("perda fetal I") os resultados foram similares, ou seja, $96,6 \%$ das mulheres com aborto induzido tiveram perda fetal e apenas $19,6 \%$ no grupo de comparação.

Como estratégia para aumentar a precisão dos coeficientes da análise multivariada, os dados do nível agregado (provenientes das mulheres alocadas no método da urna) foram transformados em dados individuais através do cálculo da probabilidade $a$ posteriori da mulher ter pelo menos um aborto induzido dado que tenha relatado perda fetal (probabilidade $a$ priori), sem afetar a confidencialidade das informações.

\section{Probabilidade de perda fetal predizer aborto induzido}

Baseando-se nas associações de aborto induzido com perda fetal foi calculada a probabilidade $a$ posteriori para cada uma das três variáveis, "perda fetal", "perda fetal $<7 \mathrm{~m}$ " e "perda fetal I" e para resposta positiva nessas três variáveis ao mesmo tempo. Para isso, utilizou-se o cálculo das verossimilhanças (sensibilidade e especificidade) de cada uma delas, as probabilidades a priori, as chances pré e pós-testes e as probabilidades $a$ posteriori das três variáveis para aborto induzido. As probabilidades a posteriori para cada uma das variáveis isoladamente foram $35,9 \%, 37,8 \%$ e $27 \%$, respectivamente para "perda fetal", "perda fetal $<7 \mathrm{~m}$ " e "perda fetal I". No entanto, observou-se que as mulheres com respostas positivas nas três variáveis relacionadas com perdas fetais tiveram uma probabilidade de $95,6 \%$ de terem induzido pelo menos um aborto. A Tabela 2 detalha todas as etapas para o cálculo da probabilidade $a$ posteriori.

A elevada probabilidade de respostas positivas nas três variáveis de história de perda fetal permitiu, com razoável segurança, que essas informações fossem utilizadas como filtro na transformação de dados do nível agregado obtidos utilizando-se o método da urna para o nível individual. Ao final dessa transformação, obteve-se 147 informações no nível individual de mulheres com relato de aborto induzido, sendo 58 provenientes do método das questões indiretas e 89 do método da urna. Nessa transformação, em 18 questionários as mulheres não preencheram os critérios de perda fetal, portanto, entre as 107 mulheres com relato de 
aborto induzido pelo método da urna, ficaram disponíveis no nível individual informações de 89 mulheres.

A Tabela 3 mostra os principais fatores de risco e preditores para as 147 mulheres com relato de aborto. $\mathrm{O}$ grupo que induziu aborto apresentou, no momento da entrevista, mais mulheres separadas/divorciadas, vivendo em união estável (morando com companheiro) e sem religião. Mais da metade das mulheres que induziram aborto não queriam ter mais filhos e tiveram problemas com métodos contraceptivos, bem como, eram mais esterilizadas e apresentavam maior conhecimento dos métodos contraceptivos existentes. Como era esperado, pelo procedimento de transformação dos dados, 99\% das mulheres com aborto induzido haviam relatado no questionário ter tido um filho que nasceu morto com menos de sete meses de gestação.

Foi realizada uma análise multivariada com a amostra total de 3.002 mulheres, incluindo todas as variáveis que haviam apresentado associação estatística com aborto na análise bivariada controlando pela idade das mulheres (Tabela 4). Após o ajuste, perderam a signifi-
Tabela 1

Associação entre relato de aborto induzido e de perda fetal para as mulheres alocadas para o método das questões indiretas $(n=1.510)$.

\begin{tabular}{|c|c|c|c|c|}
\hline \multirow[t]{3}{*}{ Variáveis para investigar } & \multicolumn{4}{|c|}{$\begin{array}{c}\text { Relato de aborto induzido } \\
\text { (método das questões indiretas) }\end{array}$} \\
\hline & \multicolumn{2}{|c|}{$\underset{(n=58)}{\operatorname{Sim}}$} & \multicolumn{2}{|c|}{$\begin{array}{c}\text { Não } \\
(n=1.452)\end{array}$} \\
\hline & $\%$ & $n$ & $\%$ & $\mathrm{n}$ \\
\hline \multicolumn{5}{|l|}{ Perda fetal* } \\
\hline Sim & 94,8 & 55 & 13,1 & 190 \\
\hline Não & 5,2 & 3 & 86,9 & 1.262 \\
\hline \multicolumn{5}{|l|}{ Perda fetal $<7 \mathrm{~m}^{\star}$} \\
\hline Sim & 94,8 & 55 & 12,0 & 175 \\
\hline Não & 5,2 & 3 & 87,9 & 1.277 \\
\hline \multicolumn{5}{|c|}{ Perda fetal I (avaliada indiretamente) } \\
\hline Sim & 96,6 & 56 & 19,6 & 284 \\
\hline Não & 3,4 & 2 & 80,4 & 1.168 \\
\hline
\end{tabular}

* Significância estatística: $p<0,001$.

Perda fetal = obtida por meio do relato de alguma gravidez com filho nascido morto; Perda fetal $<7 \mathrm{~m}=$ obtida por meio do relato de alguma perda com menos de sete meses de gravidez:

Perda fetal I = calculada por meio da subtração entre o número de gestações e o número de filhos, excluindo aqueles que faleceram após o nascimento.

\section{Tabela 2}

Verossimilhanças, probabilidade a priori, chances pré e pós-testes e probabilidade a posteriori para as três variáveis relacionadas com perda fetal.

\begin{tabular}{|c|c|c|c|c|}
\hline & Perda fetal & $\begin{array}{l}\text { Perda fetal } \\
\quad<7 \mathrm{~m}\end{array}$ & Perda fetal I & $\begin{array}{c}\text { Com as três } \\
\text { variáveis }\end{array}$ \\
\hline \multicolumn{5}{|c|}{$N=1.510$ e prevalência de aborto induzido (método das questões indiretas) $=3,8 \%$} \\
\hline Verossimilhança para teste ${ }^{+}$ & $94,8 \%$ & $94,8 \%$ & $96,6 \%$ & \\
\hline (Sensibilidade ) & $(84,7-98,7)$ & $(84,7-98,7)$ & $(87,0-99,4)$ & \\
\hline Verossimilhança para teste - & $86,9 \%$ & $87,9 \%$ & $80,4 \%$ & \\
\hline (Especificidade) & $(85,0-88,6)$ & $(86,1-89,6)$ & $(78,3-82,4)$ & \\
\hline Razão de probabilidades & 7,24 & 7,83 & 4,93 & \\
\hline \multicolumn{5}{|l|}{ (Sensibilidade/1-especificidade) } \\
\hline \multicolumn{5}{|c|}{$\mathrm{N}=1.492$ e prevalência de aborto induzido (método da urna) $=7,2 \%(0,072)$} \\
\hline Probabilidade a posteriori & $35,8 \%$ & $37,5 \%$ & $27,5 \%$ & \\
\hline$\left(\right.$ Valor preditivo $\left.{ }^{+}\right)$ & $(30,3-41,7)$ & $(31,8-43,7)$ & $(23,1-32,4)$ & \\
\hline \multicolumn{5}{|l|}{ Chance pré-teste } \\
\hline (probabilidade a priori/(1-prol & $(0,072 / 1-0,07$ & & & 0,0776 \\
\hline \multicolumn{5}{|c|}{ Chance pós-teste } \\
\hline \multicolumn{4}{|c|}{ (chance pré-teste $\times \pi$ Razões de probabilidades) $=(0,072 \times 7,24 \times 7,83 \times 4,93)$} & 21,69 \\
\hline \multicolumn{5}{|c|}{ Probabilidade a posteriori para as três variáveis de perda fetal } \\
\hline (chance pós-teste/(1+chance & $+21,69)$ & & & $95,6 \%$ \\
\hline
\end{tabular}

Perda fetal = obtida por meio do relato de alguma gravidez com filho nascido morto;

Perda fetal $<7 \mathrm{~m}=$ obtida por meio do relato de alguma perda com menos de sete meses de gravidez;

Perda fetal I = calculada por meio da subtração entre o número de gestações e o número de filhos,

excluindo aqueles que faleceram após o nascimento. 
Comparação das características demográficas e reprodutivas das 147 mulheres que induziram aborto com o restante da amostra $(n=3.002)$.

\begin{tabular}{|c|c|c|c|c|c|}
\hline \multirow{3}{*}{$\begin{array}{l}\text { Características com diferenças } \\
\text { significativas }\end{array}$} & \multicolumn{4}{|c|}{ Relato de aborto induzido } & \\
\hline & \multicolumn{2}{|c|}{ Não induziram $(n=2.855)$} & \multicolumn{2}{|c|}{ Induziram $(n=147)$} & \\
\hline & $\%$ & $\mathrm{n}$ & $\%$ & $\mathrm{n}$ & \\
\hline \multicolumn{6}{|l|}{ Estado civil } \\
\hline Casada & 47,2 & 1.348 & 47,6 & 70 & $p=0,001$ \\
\hline Em união & 9,1 & 259 & 15,6 & 23 & \\
\hline Separada/Divorciada & 6,7 & 192 & 12,2 & 18 & \\
\hline Solteira & 34,9 & 997 & 23,1 & 34 & \\
\hline Viúva & 2,1 & 59 & 1,4 & 2 & \\
\hline Mora com companheiro & 10,4 & 297 & 18,4 & 27 & $p=0,002$ \\
\hline \multicolumn{6}{|l|}{ Religião } \\
\hline Católica & 59,9 & 1.710 & 44,9 & 66 & $p<0,001$ \\
\hline Protestante & 13,2 & 377 & 12,2 & 18 & \\
\hline Espírita & 11,1 & 318 & 19,7 & 29 & \\
\hline Outras & 6,4 & 184 & 10,9 & 16 & \\
\hline Não tem religião & 9,3 & 266 & 12,2 & 18 & \\
\hline Não quer mais filhos & 49,1 & 1.402 & 66,0 & 97 & $p<0,001$ \\
\hline $\begin{array}{l}\text { Perdas (número de gestações - } \\
\text { número de nascidos vivos)* }\end{array}$ & 18,9 & 539 & 90,5 & 133 & $p<0,001$ \\
\hline \multicolumn{6}{|l|}{ Filho que nasceu morto* } \\
\hline Sim & 12,8 & 366 & 88,4 & 130 & $p<0,001$ \\
\hline$<7$ meses de gestação & 89,9 & 329 & 99,2 & 129 & $p<0,001$ \\
\hline$\geq 7$ meses de gestação & 10,1 & 37 & 0,8 & 1 & \\
\hline Esterelizadas & 10,2 & 290 & 17,0 & 25 & $p=0,008$ \\
\hline Teve problemas com método contraceptivo & 36,8 & 866 & 51,1 & 71 & $p<0,001$ \\
\hline $\begin{array}{l}\text { Conhecimento de contraceptivos } \\
\text { (número médio de métodos) }\end{array}$ & & 3,4 & & 3,8 & $p<0,001$ \\
\hline
\end{tabular}

* Variáveis utilizadas na construção do desfecho final de aborto induzido ( $n=147)$.

cância estatística as variáveis referentes ao desejo de ter mais filhos e esterilização. Mulheres vivendo em união consensual apresentaram um risco de induzir um aborto cerca de duas vezes maior comparado com as mulheres casadas. Esse fato pode ser confirmado pela variável "mora com companheiro", que apresentou um risco três vezes maior. Não ter religião aumentou o risco de aborto em $100 \%$ comparado com as mulheres de religião católica. Conhecer um maior número de métodos contraceptivos mostrou um aumento no risco de $50 \%$ para aborto, mesmo após o controle para idade. Ainda na Tabela 4, pode ser observado um OR = 1,50 (IC95\%: 1,1-2,1) para as mulheres que tiveram problemas com métodos contraceptivos.
Para a melhor compreensão da dinâmica dos fatores de risco e preditores do aborto induzido segundo as diferentes etapas das mulheres durante a vida reprodutiva, foram realizadas análises estratificadas por grupo etário. Entre as 147 mulheres com aborto, oito tinham idade de 15 a 19 anos, 27 mulheres de 20 a 29 anos, 51 de 30 a 39 e as 61 restantes de 40 a 49 . Para a menor faixa de idade (15 a 19 anos), o número de abortos foi muito pequeno inviabilizando a realização de testes de associação com aborto - possibilitando, apenas, observarse tendências.

A Tabela 5 apresenta, por faixa etária, um resumo dos principais preditores presentes na história de vida das mulheres, que apontam 
para o risco de praticar um aborto. Para as adolescentes os principais preditores foram ser de famílias de baixa renda e estarem afastadas da escola; entre a idade de 20 e 29 anos, estarem separadas ou divorciadas; para a idade de 30 a 39 anos, viverem com companheiro, isto é, em união, terem tido problemas com métodos contraceptivos, terem maior conhecimento dos métodos existentes e serem esterilizadas. $\mathrm{Na}$ última faixa etária, de 40 a 49 anos, embora um maior número de mulheres tenha pelo menos induzido um aborto em sua vida, observou-se uma homogeneidade de exposição, isto é, praticamente todas as mulheres vivenciaram as diversas exposições ou situações de vida reprodutiva testadas neste estudo.

\section{Discussão}

Em epidemiologia, um fator de risco pode ter dois significados principais: (a) um atributo ou exposição que aumenta a probabilidade de ocorrência de doença ou outro desfecho específico, sendo dito como determinante e (b) um atributo ou exposição associada com o aumento da probabilidade de ocorrência de doença ou outro desfecho específico, não necessariamente como um fator causal, sendo denominado um marcador, preditor ou indicador de risco 10,11 .

O desenho transversal utilizado neste estudo não assegura a verificação da temporalidade entre a causa e o efeito, isto é, entre os fatores de risco e a ocorrência de aborto induzido. Por esse motivo, as características que apresentaram associação com aborto devem ser vistas como preditores, mesmo que, em algum momento na vida das mulheres, possam ter sido reais fatores de risco. Além do mais, neste estudo foi considerada a indução do aborto em qualquer momento da vida reprodutiva, com possibilidade de mais de um evento por mulher, o que contribui para a ocorrência de causalidade reversa. O maior conhecimento de métodos contraceptivos por parte das mulheres que induziram aborto pode ser conseqüente a esse viés.

Usualmente, estudos sobre aborto induzido exigem criatividade e combinação de metodologias, tanto para a coleta de dados, bem como na estimativa de suas taxas 6,12. Nosso estudo foi inovador na medida em que para a análise e interpretação dos dados foram utilizados métodos estatísticos epidemiológicos e a aplicado o Teorema de Bayes para determinação das probabilidades $a$ posteriori. A análise freqüentista, utilizada na grande maioria dos es-
Tabela 4

Análise multivariada (regressão logística) das variáveis associadas com aborto induzido, ajustadas para idade das mulheres $(n=3.002)^{*}$.

\begin{tabular}{|c|c|c|c|}
\hline Características & OR & IC95\% & $p$ valor \\
\hline \multicolumn{4}{|l|}{ Estado civil } \\
\hline Casada & 1,00 & & \\
\hline Em união & 2,07 & $1,21-3,53$ & $p=0,015$ \\
\hline Separada/Divorciada & 1,85 & $1,13-3,04$ & \\
\hline Solteira & 1,50 & $0,85-2,64$ & \\
\hline Viúva & 0,30 & $0,04-2,25$ & \\
\hline \multicolumn{4}{|c|}{ Mora com companheiro** } \\
\hline Não & 1,00 & & \\
\hline Sim & 3,15 & $1,56-6,38$ & $p=0,01$ \\
\hline \multicolumn{4}{|l|}{ Religião } \\
\hline Católica & 1,00 & & \\
\hline Protestante & 1,32 & $0,75-2,30$ & $p=0,02$ \\
\hline Espírita & 1,71 & $1,05-2,78$ & \\
\hline Outras & 2,21 & $1,19-4,11$ & \\
\hline Não tem religião & 2,00 & $1,14-3,51$ & \\
\hline \multicolumn{4}{|l|}{ Quer mais filhos } \\
\hline Sim & 1,00 & & \\
\hline Não & 1,08 & $0,69-1,69$ & $p=0,744$ \\
\hline \multicolumn{4}{|l|}{ Esterelizadas } \\
\hline Não & 1,00 & & \\
\hline Sim & 0,88 & $0,54-1,43$ & $p=0,600$ \\
\hline \multicolumn{4}{|c|}{$\begin{array}{l}\text { Teve problema com método } \\
\text { contraceptivo }\end{array}$} \\
\hline Não & 1,00 & & \\
\hline Sim & 1,50 & $1,05-2,14$ & $p=0,025$ \\
\hline \multicolumn{4}{|c|}{ Conhecimento de contraceptivos } \\
\hline$\leq 3$ métodos & 1,00 & & \\
\hline$\geq 4$ métodos & 1,55 & $1,08-2,22$ & $p=0,017$ \\
\hline
\end{tabular}

* As medidas para as três variáveis medindo perda fetal não são mostradas, uma vez que foram utilizadas na construção do desfecho final de aborto induzido $(n=147)$;

** A variável mora com companheiro foi analisada em um modelo independente de estado civil.

tudos epidemiológicos, lida com a probabilidade de um conjunto de dados a partir de uma hipótese. Por outro lado, utilizar o teorema de Bayes empírico diz respeito à probabilidade de que uma hipótese, ou uma conclusão, seja verdadeira frente aos dados observados 13.

Utilizando-se da informação de que perda fetal foi o fator que esteve mais fortemente associado ao aborto induzido (informações $a$ priori), foi possível o cálculo da probabilidade a posteriori. Com isso, pode ser observado que as mulheres com respostas positivas nas três 
Principais preditores de aborto induzido por grupo de idade.

\begin{tabular}{|c|c|c|c|}
\hline \multicolumn{4}{|c|}{ Grupos etários (anos) } \\
\hline 15 a $19^{*}$ & 20 a 29 & 30 a 39 & 40 a 49 \\
\hline & $\begin{array}{l}\text { Separadas ou divorciadas } \\
(p=0,06)\end{array}$ & $\begin{array}{l}\text { Vivem em união } \\
(p=0,04)\end{array}$ & \\
\hline & & $\begin{array}{l}\text { Moram com companheiros } \\
(p<0,001)\end{array}$ & \\
\hline \multicolumn{4}{|l|}{$\begin{array}{l}\text { Provêm de famílias } \\
\text { com menor renda }\end{array}$} \\
\hline \multicolumn{4}{|l|}{$\begin{array}{l}\text { Não estudam ou têm } \\
\text { baixa escolaridade }\end{array}$} \\
\hline & & $\begin{array}{l}\text { Não têm religião } \\
(p=0,03)\end{array}$ & $\begin{array}{l}\text { São espíritas } \\
(p=0,01)\end{array}$ \\
\hline \multirow[t]{3}{*}{$\begin{array}{l}\text { Conhecem em média } 3,5 \\
\text { métodos contraceptivos }\end{array}$} & & $\begin{array}{l}\text { Conhecem em média } 4,0 \\
\text { métodos contraceptivos } \\
(p<0,001)\end{array}$ & $\begin{array}{l}\text { Conhecem em média } 3,6 \\
\text { métodos contraceptivos } \\
(p=0,02)\end{array}$ \\
\hline & & $\begin{array}{l}\text { Tiveram problemas com } \\
\text { métodos contraceptivos } \\
(p=0,08)\end{array}$ & \\
\hline & & $\begin{array}{l}\text { Fizeram esterilização } \\
(p<0,001)\end{array}$ & \\
\hline Relato de perda fetal & $\begin{array}{l}\text { Relato de perda fetal } \\
(p<0,001)\end{array}$ & $\begin{array}{l}\text { Relato de perda fetal } \\
(p<0,001)\end{array}$ & $\begin{array}{l}\text { Relato de perda fetal } \\
(p<0,001)\end{array}$ \\
\hline
\end{tabular}

* Para a faixa etária de 15 a 19 anos não foram realizados testes estatísticos de associação devido ao número reduzido de relato do aborto induzido. Estão sendo apresentadas as características mais freqüentes.

variáveis relacionadas com perdas fetais tinham uma probabilidade de $95,6 \%$ de terem induzido pelo menos um aborto. Essa alta probabilidade permitiu a passagem de dados agregados para individuais e, conseqüentemente, aumentar a precisão na análise multivariada.

Quanto ao achado de perda fetal ser fator de risco ou preditor para aborto induzido, levanta-se duas possibilidades. A primeira seria uma associação causal entre perda fetal "espontânea" - uma vez que a pergunta utilizada dava a idéia de aborto espontâneo - e história de aborto induzido. Esse resultado é consistente com achados de outros estudos, demonstrando um risco aumentado para aborto espontâneo em gravidez subseqüente a um aborto induzido 14,15 . A outra possibilidade para a principal associação encontrada em nosso estudo (perda fetal $x$ aborto induzido) que deve ser considerada, seria o desejo das mulheres de alguma maneira, mesmo que sutil, relatarem a experiência de aborto induzido. A pergunta de perda fetal era feita na primeira parte da entrevista, onde a princípio, as mulheres desconheciam que seriam abordadas sobre questões de aborto induzido. Essa hipótese poderia ser apoiada pelo achado no estudo de Magnani et al. 16, onde foi encontrado um grande número de casos de perda fetal, relatados pelas mulheres como espontâneos, mas classificados, pelo algorítimo proposto no estudo, como induzido. Cabe salientar que os valores para perda fetal encontrados no presente estudo foram superiores aos apresentados por Casterline 17 para quarenta países - o próprio autor enfatizou que as estimativas estavam aquém do esperado. Certamente devem ser realizados outros estudos com acompanhamento longitudinal, talvez combinados com metodologias qualitativas, para melhor entender esse achado.

Embora tenha sido estudado o efeito de todas as variáveis sócio-econômicas, demográficas e reprodutivas presentes no questionário geral, que pudessem ser possíveis fatores de risco ou preditores para o aborto induzido, poucas variáveis mostraram-se associadas. Nenhuma das variáveis sócio-econômicas apresentou associação significativa com aborto, exceto a tendência encontrada no grupo das adolescentes. Ao observar o conjunto das mulheres, o estudo mostrou que aborto esteve associado com as mulheres que viviam em união ou estavam separadas/divorciadas no momento da entrevista. Resultados de outros estudos mostram 
achados similares 18, embora em alguns desses apresentem diferenças na categorização da variável estado civil 19,20. As mulheres com relato de aborto tinham feito mais esterilização, não queriam mais filhos, tiveram problemas com algum método contraceptivo e em média conheciam mais métodos contraceptivos do que as mulheres que não abortaram. A possibilidade de esterilização é relacionada ao uso anterior de métodos contraceptivos, particularmente as falhas e efeitos adversos dos métodos 21 . $\mathrm{O}$ aborto foi menos prevalente em mulheres católicas. Na análise multivariada, controlando esses efeitos para idade das mulheres, desapareceu a associação com a esterilização.

Quando a análise foi estratificada pela idade, as adolescentes que relataram aborto apresentaram algumas características específicas. Entre as oito meninas, $50 \%$ delas relataram não usar método contraceptivo e já terem filhos. Embora a maioria das adolescentes da amostra total fosse católica, o inverso foi observado para as adolescentes que haviam relatado aborto. Apenas duas das adolescentes estavam na escola e dois terços referiram que sua atividade era ser dona-de-casa ou não tinham atividade. Este fato refletiu na escolaridade média desse grupo, 5,9 anos enquanto que, para essa faixa etária, espera-se uma escolaridade média entre oito e nove anos (oito anos do ensino fundamental e pelo menos um do ensino médio). A renda mensal das famílias das adolescentes com relato de aborto foi significativamente menor do que a das outras adolescentes. Esses resultados são compatíveis com os apresentados por Wartenberg 22 para adolescentes de baixo nível sócio-econômico, tais como o afastamento da escola, a existência de outros filhos e inatividade - aumentando o risco de uma gestação não desejável por incapacidade de canalizar suas vidas de forma produtiva. A relação de pobreza com aborto parece ser uma característica apenas observada entre as adolescentes.

Como descrito acima, as adolescentes que induziram aborto parecem apresentar piores condições sócio-econômicas do que as outras meninas da mesma idade. Durante interpretação desse resultado também deve ser conside- rada a possibilidade de relato diferenciado para as adolescentes conforme a classe social, isto é, meninas com melhores condições de vida escondem - não relatam - suas experiências de aborto. Para os outros grupos etários, renda familiar e escolaridade não estiveram associadas ao aborto, podendo sugerir a ocorrência, ou relato, de aborto similar entre as camadas sociais.

Para o grupo etário de 20 a 29 anos de idade, o principal preditor foi estar separada ou divorciada. Esse achado é consistente com o estudo de Sihvo et al. 23, que mostra um risco aumentado de induzir um aborto frente a uma gravidez não desejada em mulheres com relacionamentos não estáveis. Para o próximo grupo de mulheres, 30 a 39 anos, mais características estiveram associadas, como viverem em união; não terem religião; serem esterilizadas; terem mais problemas com métodos contraceptivos e, conseqüentemente, conhecerem mais métodos.

Na última faixa etária houve uma homogeinização, isto é, ao final da vida reprodutiva as mulheres já tiveram oportunidade de ser expostas às mais diversas situações, dificultando a identificação de riscos por meio da abordagem transversal utilizada neste estudo. Mesmo assim, foram encontradas associações significativas de religião e conhecimento de métodos contraceptivos com aborto.

Finalmente, o sub-relato de aborto induzido em pesquisas é inerente ao tema, principalmente quando abordado em estudos de base populacional. Neste estudo, por meio da combinação de metodologias foi possível traçar o perfil das mulheres em risco de submeteremse ao aborto. Mesmo que os resultados mostrem o aborto sendo realizado independente das características sócio-econômicas e, inclusive, das características étnicas das mulheres, sabe-se que aquelas pertencentes a classes sociais menos favorecidas são expostas a procedimentos abortivos inseguros 3 . Em nossos resultados, as características reprodutivas e o estado conjugal das mulheres foram os maiores fatores de risco e preditores para o aborto induzido. Portanto, espera-se que essas informações subsidiem o planejamento de ações e políticas públicas na área de saúde reprodutiva. 


\section{Resumo}

O presente trabalho investigou os principais fatores de risco e preditores para o aborto induzido. Foi realizado um estudo transversal de base populacional com uma amostra representativa de 3.002 mulheres de 15 a 49 anos residentes no Sul do Brasil. Para responder as questões de aborto, as mulheres foram alocadas entre duas metodologias: método da urna ou método das questões indiretas. Informações sócio-econômicas, demográficas e reprodutivas foram obtidas por meio de um questionário pré-codificado. Na análise e interpretação dos dados utilizou-se o modelo de regressão logística. Teorema de Bayes foi aplicado para a determinação das probabilidades a posteriori permitindo a transformação dos dados agregados em dados individuais. Abortos induzidos estiveram fortemente relacionados com relatos de perda fetal em todas as idades. Entre as adolescentes, os principais preditores foram: pertencer a famílias de baixa renda, ter baixa escolaridade e alta evasão escolar, além de conhecerem um número maior de métodos contraceptivos. Para as mulheres de 20 a 49 anos de idade não houve diferença sócio-econômica, sendo que, estado civil e características reprodutivas, incluindo conhecimento de métodos contraceptivos, foram os fatores de risco freqüentes para o aborto induzido.

Saúde da Mulher; Aborto Induzido; Fatores de Risco

\section{Referências}

1. World Health Organization. Complications of abortion: technical and managerial guidelines for prevention and treatment. Geneva: World Health Organization; 1995.

2. United Nations. The world's women 1995: trends and statistics. New York: United Nations; 1995.

3. Paxman J, Rizo A, Brown L, Benson J. The clandestine epidemic: the practice of unsafe abortion in Latin America. Stud Fam Plann 1993; 24:20526.

4. Ross J, Frankenberg E. Findings from two decades of family planning research. New York: Population Council; 1993.

5. Guedes AC. Abortion in Brazil: legislation, reality and options. Reprod Health Matters 2000; 8:6676.

6. Rossier C. Estimating induced abortion rates: a review. Stud Fam Plann 2003; 34:87-102.

7. Olinto MTA, Moreira-Filho D. Estimativa de aborto induzido: comparação entre duas metodologias. Rev Panam Salud Pública 2004; 15:331-6.

8. Huntington D, Mensch B, Toubia N. A new approach to eliciting information about induced abortion. Stud Fam Plann 1993; 24:120-4.

\section{Colaboradores}

M. T. Olinto trabalhou em todas as etapas até a finalização deste artigo. D. C. Moreira-Filho orientou todas as etapas da análise e colaborou na redação do artigo.

\section{Agradecimentos}

Estudo financiado pelo Population Council (escritório de Nova York, Estados Unidos) e pelo Conselho Nacional de Desenvolvimento Científico e Tecnológico (CNPq) (processo n. 142783/96-0).
9. Olinto MTA. Estimativa da freqüência de abortos induzidos: teste de uma nova metodologia. Revista Brasileira de Estudos Populacionais 1994; 11:255-8.

10. Norell S. A short course in epidemiology. New York: Raven; 1992.

11. Last J. A dictionary of epidemiology. Oxford: Oxford University Press; 1988.

12. Coeytaux F. Methodological issues in abortion research. New York: Population Council; 1989.

13. Ashby D, Hutton JL. Bayesian epidemiology. Berry DA, Stangl DK, editors. Bayesian biostatistics. New York: Marcel Dekker Inc.; 1996. p. 109-38.

14. Zhou W, Olsen J, Nielsen GL, Sabroe S. Risk of miscarriage following induced abortion is only increased with short interpregnancy interval. J Obstet Gynaecol 2000; 20:49-54.

15. Sun Y, Che Y, Gao E, Olsen J, Zhou W. Induced abortion and risk subsequent miscarriage. Int $\mathrm{J}$ Epidemiol 2003; 32:449-54.

16. Magnani RJ, Rutenberg N, McCann HG. Detecting induced abortions from reports of pregnancy terminations in DHS calendar data. Stud Fam Plann 1996; 27:36-43. 
17. Casterline J. Collecting data on abortion using national surveys: methodological issues in abortion research. New York: Population Council; 1989.

18. Silva RS. Patterns of induced abortion in urban area of Southeastern region, Brazil. Rev Saúde Pública 1998; 32:7-17.

19. Fonseca W, Misago C, Correia LL, Parente JAM, Oliveira FC. Determinantes de aborto provocado entre mulheres admitidas em hospitais em localidade da região Nordeste do Brasil. Rev Saúde Pública 1996; 30:13-8.

20. Schor N. Investigação sobre a ocorrência de aborto em pacientes de hospital de centro urbano do Estado de São Paulo, Brasil. Rev Saúde Pública 1990; 24:144-51.
21. Vieira EM. Do women's attitudes towards abortion and contraceptive methods influence their option for sterilization? Cad Saúde Pública 1999; 15:739-47.

22. Wartenberg L. El aborto en Colombia: factores asociados derivados del contexto familiar y de la relación de pareja. In: Encuentro de Investigadores sobre el Aborto Inducido en América Latina e Caribe. Determinantes del Aborto y Factores Asociados. Colombia: Universidad Externado de Colombia; 1994.

23. Sihvo S, Bajos N, Ducot B, Kaminski M; Cocon Group. Women's life cycle and abortion decision in unintended pregnancies. J Epidemiol Community Health 2003; 57:601-5.

Recebido em 10/Mai/2004

Versão final reapresentada em 28/Jun/2005

Aprovado em 19/Jul/2005 